\title{
The brain's past
}

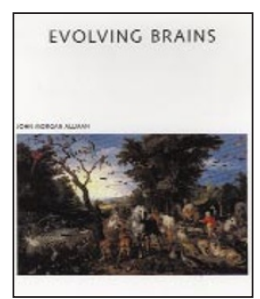

Humans share a desire to explain our place in nature and through such explanations to make sense of our unique role on earth. What makes us different? Not just our intellect, because many species have an enviable intelligence evolved for their particular lives. Clearly what sets us apart is our cognitive capacities, particularly our linguistic skills, our power of introspection and the 'big C', consciousness. So, from the bushy human tree, how did we develop such remarkable capacities? In his new book, Evolving Brains, John Allman, a distinguished comparative neuroscientist, takes on this fundamental question from the perspective of brain evolution. Can we understand our phylogenetic success through the lens of brain evolution?

What needs to be explained? Protohumans diverged from protochimpanzees some 6 million years ago, and the genus Homo emerged about 2.5 million years ago, coincident with the oldest archeological sites showing artifact manufacture and carnivory. About a million years later, Homo ergaster appeared, our first exclusively bipedal ancestor, able to invade seasonal environments. Yet paleontological evidence shows something really important occurred around 50,000 years ago, when fully modern humanoids emerged and spread out of Africa. Homo heidelbergensis was our successful precursor, and coincident with her appearance, archaeologists have collected a laundry list of unique attributes, including spatial organization of camps, transport of raw materials for great distances, elaborate graves, colonization of the inhospitable climates of northeastern Europe and northern Asia, fishing and the appearance of art.

At about this time, the rate of behavioral change clearly accelerated, probably because our ancestors evolved the capacity to invent, and innovation became a regular feature of life. What might explain this sudden success

Russell Fernald is at the Department of Psychology, Stanford University, Bldg. 420, Rm. 436, Stanford, California 94305-2310, USA. e-mail: russ@psych.stanford.edu
50,000 years ago? Paleoneurologists claim a fortuitous mutation promoted the modern brain, and this neural substrate allowed biological and culural evolution to accelerate. Certainly, increased brain size would be a an obvious explanation, but estimated brain size has been increasing almost exponentially, beginning about 500,000 years ago, well before documented behavioral changes. Moreover, brain functions are diffusely distributed, so brain size alone cannot be the answer, particularly because brain evolution is not tidy but results from adding to, modifying and reorganizing existing structures. What then might be an explanation?

Allman offers a number of plausible reasons why we became the only species that can read books, much less write them. One of his central themes is that duplication was fundamental in the evolution of brains, just as it is in development. Gene duplication, thought to be responsible for the rise of vertebrates, could also explain multiple sensory maps, multiple kinds of hair cells that enhance hearing, multiple genes responsible for construction of the cortex and other important brain structures. Gene duplications could provide a substrate for diversification of structures that would expand the brain's repertoire.

In a beautifully illustrated part of the book, Allman traces the evolution of warmblooded animals, weaving together modern neuroscientific findings with anthropological data. The high cost of big brains (about $20-25 \%$ of total energy whether awake or asleep) is possible because thermoregulation evolved. Thus, despite its cost, expanding the neocortex must have been worth it, but exactly what it brought early man is not clear. For each apparent evolutionary advantage such as frontal vision, thought to have evolved for moving rapidly in trees, there is an exception. Squirrels, with lateral eyes, for example, do just fine in trees. Allman present all sides of each argument in thoughtful, clear prose, and it is a delight to find interesting jewels throughout the book.

In the discussion of primate brains, Allman is particularly insightful. He describes how the visual system might work, including how faces, motion, size and color could be encoded. He elucidates evolutionary forces that led to brain specializations as well. For example, fruit-eating primates have distinctly different brain structures and larger relative brain sizes than leaf-eating primates. This kind of information should inspire neuroscientists to step back from their electrodes (or gels) and think about how evolutionary forces might have shaped the system they are probing.

Throughout the book, Allman is clear about what has not worked as an explanation for brain evolution. For example, the social setting has often been invoked as an evolutionary imperative that produced larger brains. Yet, the 'Machiavellian' hypothesis, that it takes a mastery of intrigue associated with a renaissance prince to succeed in primate society, is not supported by brain size measurements. Indeed, longevity seems to be more strongly correlated with features of brain structure.

Anyone who has thought about what may have launched $H$. sapiens has a favorite. For Allman, this is the domestication of dogs. Although I like dogs, this scenario seems improbable for several reasons. It is unlikely that any single event propelled H. sapiens to the top. Besides, even though DNA data puts dog domestication at about 135,000 years ago, osteological data put the event only 14,000 years ago. Moreover, the geographical distribution of wolves did not extend to Africa at the time DNA dating suggests domestication occurred. Thus the human and dog cooperation as portrayed probably could not have been the key event.

Dog domestication aside, Evolving Brains is a wonderful read. In turns thoughtful and challenging, it is a wellwoven fabric of insights about brain evolution. I would have welcomed Allman's thoughts about some of the larger issues that surround brain science. How does a brain create a mind, and what other species might have minds. And, of course, what about consciousness? Why and how did it evolve? Is our state of being able to look inward unique? Do the exquisite brains of other primates described here have consciousness or something like it?

Ultimately, the events responsible for the great leap forward 50,000 years ago may turn out to be quite unremarkable. Perhaps it was the appearance of a new receptor family, vesicle-release protein or neurotransmitter type, untraceable in the fossil record. Compared with its consequences, the original causes might have been very small events indeed. 